\title{
Correct Labeling in Multiple Pregnancy
}

\author{
Arushi Yadav $^{1} \cdot$ Jogender Kumar $^{2}$ (D)
}

Received: 14 September 2019 / Accepted: 21 November 2019 / Published online: 20 December 2019

(C) Dr. K C Chaudhuri Foundation 2019

To the Editor: The procedure for labeling twins differs in the antenatal and postnatal period. Antenatally, the twins are labeled T1/T2 as per their relationship with cervical canal. However, postnatally, they are labeled as per their birth order [1]. A perinatal switch in vaginal birth may occur if $\mathrm{T} 2$ delivers through a fold of the intertwin membrane [2]. On the other hand, in cesarean deliveries this switch is frequent. This variation in nomenclature leads to confusion among healthcare providers and makes the twins prone to medical errors [3]. Also, it may introduce mistrust in the doctorpatient relationship. Here we describe two such cases.

Case 1: A pair of twins were born at 36 wk of gestation by cesarean section. Antenatal scan at $32 \mathrm{wk}$ showed posterior fossa cyst in one of the twins (labeled as T2). Follow-up postnatal scan was done for the baby born second in the birth order (labeled as T2 by a pediatrician) and was reported normal. It created confusion and feeling of mistrust among parents for the radiologist who did the antenatal scan. On follow-up, they consulted another pediatrician and discussed this discrepancy. $\mathrm{He}$ advised ultrasound of another infant (labeled as T1 at birth), and posterior fossa cyst was visualized in this infant.

Case 2: Another pair of twins were born at $34 \mathrm{wk}$ by vaginal delivery. Antenatal scan at 29 wk showed moderate hydronephrosis of kidneys in T1. The pediatrician ordered follow-up ultrasound for the twin born first in the order, and it was reported normal. Both the scans were done by the same radiologist, so she raised doubt and asked for another twin's scan. The scan of twin born second in order showed hydronephrosis. The parents had a conflict with the pediatrician.

Jogender Kumar

jogendrayadv@gmail.com

1 Department of Radiodiagnosis, Government Medical College and Hospital, Chandigarh, India

2 Department of Pediatrics, Post Graduate Institute of Medical Education and Research, Chandigarh 160012, India
Such scenarios create confusion leading to unnecessary investigations and breach in the doctor-patient trust. Such confusion in critical cases like duct-dependent cardiac lesion may be fatal. None of the suggested methods to avoid these errors are accurate $[1,2,4,5]$. Therefore, the most practical solution is screening of both twins using non-invasive methods like ultrasonography before declaring them normal. The awareness among healthcare providers about this perinatal switch and difference in the method of labeling may avoid medical errors. The antenatal counseling of the parents should be done by stating that one of the babies has this problem rather than addressing them as T1/T2. They should be explained about the need of postnatal screening for both the babies. It will help in strengthening the doctor-patient relationship.

\section{Compliance with Ethical Standards}

Conflict of Interest None.

\section{References}

1. Khalil A, Rodgers M, Baschat A, et al. ISUOG practice guidelines: role of ultrasound in twin pregnancy: ISUOG guidelines. Ultrasound Obstet Gynecol. 2016;47:247-63.

2. Dias T, Ladd S, Mahsud-Dornan S, Bhide A, Papageorghiou AT, Thilaganathan B. Systematic labeling of twin pregnancies on ultrasound. Ultrasound Obstet Gynecol. 2011;38:130-3.

3. Adelman JS, Applebaum JR, Southern WN, et al. Risk of wrongpatient orders among multiple vs singleton births in the neonatal intensive care units of 2 integrated health care systems. JAMA Pediatr 2019. Available at: https://jamanetwork.com/journals/ jamapediatrics/fullarticle/2748683. Accessed 13 Sep 2019.

4. Kontopoulos E, Quintero R, Barrett J, Chmait R. Which twin is which? A proposed solution for the labeling of twins at birth. Am J Obstet Gynecol. 2015;213:245-6.

5. Freed GL. When new standards to improve safety do not actually improve safety. JAMA Pediatr. 2019;173:921-2.

Publisher's Note Springer Nature remains neutral with regard to jurisdictional claims in published maps and institutional affiliations. 